\title{
Profissão nutricionista: 70 anos de história no Brasil
}

\author{
Profession of dietician: 70 years \\ of history in Brazil
}

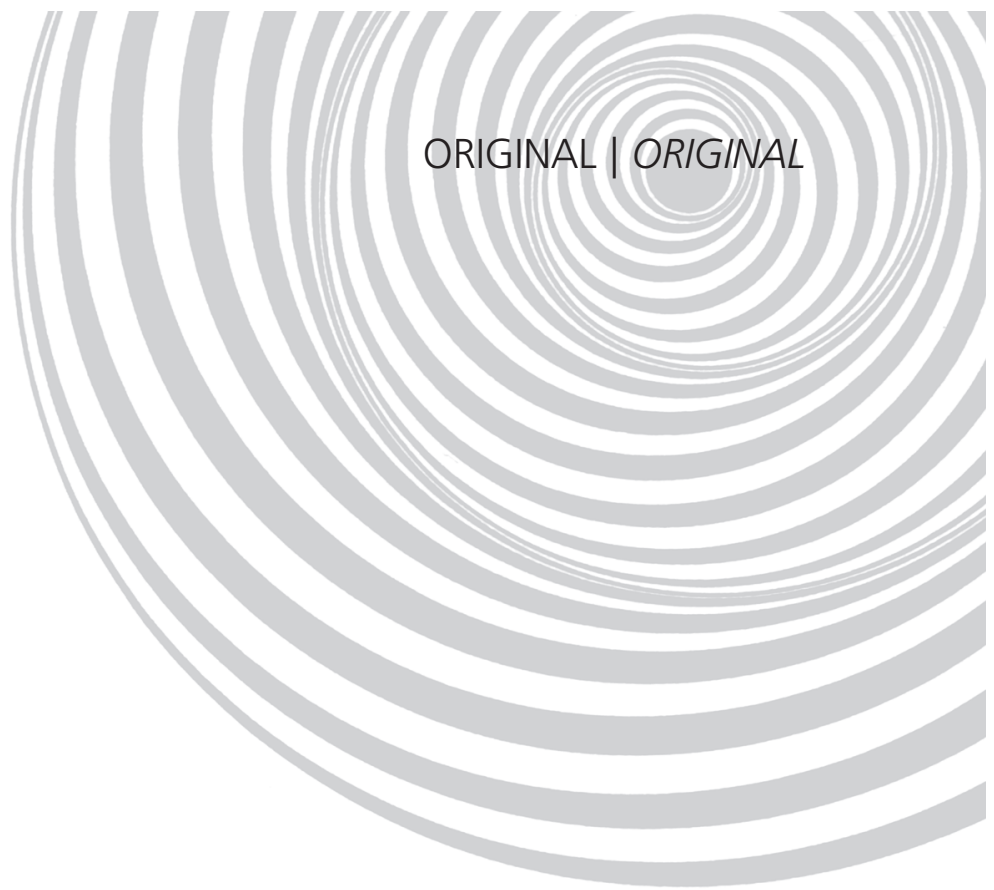

Francisco de Assis Guedes de VASCONCELOS'

Carmen Lúcia de Araújo CALADO²

RE S U M O

\section{Objetivo}

Realizar análise histórica dos 70 anos da profissão de nutricionista no Brasil, procurando analisar a expansão do número de cursos de graduação em nutrição e de nutricionistas no país e a identidade do nutricionista brasileiro no contexto atual.

\section{Métodos}

A base empírica foi constituída pelas estatísticas do número de profissionais e de cursos disponibilizadas pelo Conselho Federal de Nutricionistas e pelos dados da pesquisa "Inserção Profissional dos Nutricionistas no Brasil", realizada em 2005, pelo Conselho Federal de Nutricionistas.

\section{Resultados}

Em agosto de 2009, existiam 391 Cursos de Nutrição no Brasil (324 privados e 67 públicos), com 49185 vagas anuais. Em junho de 2009, existiam 60554 nutricionistas registrados no País, perfazendo uma relação de um nutricionista para cada 3162 habitantes. De acordo com pesquisa do Conselho Federal de Nutricionistas, em 2005, o nutricionista brasileiro apresentava o seguinte perfil: 96,5\% do sexo feminino; 79,4\% entre 20 e 40 anos; $53,6 \%$ solteiros; $79,1 \%$ de cor branca; $41,7 \%$ atuando em nutrição clínica; 32,2\% em alimentação coletiva; 9,4\% em ensino (docência); 8,8\% em saúde coletiva; 4,1\% em nutrição esportiva e 3,7\% em indústria de alimentos.

\section{Conclusão}

A análise aponta intensa elevação do número de cursos e de profissionais e diversificação das áreas de atuação do nutricionista. Além das sete grandes áreas de atuação reconhecidas pelo Conselho Federal de Nutricionistas, observa-se dentro delas um amplo processo de divisão dos seus objetos de trabalho. É provável que, nos próximos anos, em função do lançamento de maior número de profissionais, ocorra uma redistribuição geográfica dos nutricionistas, modificações no mercado de trabalho e áreas de atuação profissional.

Termos de indexação: Ciência da nutrição. Nutricionista. Ocupações em saúde.

\footnotetext{
1 Universidade Federal de Santa Catarina, Centro de Ciências da Saúde, Departamento de Nutrição. Campus Universitário, Trindade. 88040-9000, Florianópolis, SC, Brasil. Correspondência para/Correspondence to: F.A.G. VASCONCELOS. E-mail: <fguedes@floripa.com.br>

2 Universidade Federal do Rio Grande do Norte, Ouvidora. Natal, RN, Brasil.
} 
606 | F.A.G. VASCONCELOS \& C.L.A. CALADO

\section{A B S T R A C T}

\section{Objective}

This study made a historical analysis of the 70 years of the profession of dietician in Brazil, attempted to analyze the increase in the number of undergraduate nutrition courses and dieticians in the country and analyzed the identity of the Brazilian dietician in the current context.

\section{Methods}

The empirical basis was based on the number of professionals and courses offered by the Federal Council of Dieticians and on the Federal Council of Dieticians research Insertion of Professional Dieticians in Brazil, done in 2005.

\section{Results}

There were 391 courses of nutrition in Brazil (324 private and 67 public), enrolling 49, 185 students yearly in August 2009. In June 2009, there were 60,554 registered dieticians in the country, resulting in a ratio of one dietician per 3,162 inhabitants. According to the 2005 Federal Council of Dieticians' research, Brazilian dieticians have the following profile: $96.5 \%$ are females, $79.4 \%$ aged 20 to 40 years, $53.6 \%$ are single, $79.1 \%$ are Caucasian, $41.7 \%$ were working in clinical nutrition, $32.2 \%$ in food services, $9.4 \%$ in academia, $8.8 \%$ in public health, $4.1 \%$ in sports nutrition and $3.7 \%$ in the food industry.

\section{Conclusion}

The analysis shows a steep increase in number of courses and professionals and diversification of the dietician's areas of expertise. Additionally, within the seven major areas of expertise recognized by the Federal Council of Dieticians, there is a major process of division of their work objects. It is likely that changes in the labor market and work areas will take place in the near future because of the greater number of professionals in the market and their geographical redistribution.

Indexing terms: Nutritionist. Health occupations. Nutritional.

\section{N T R O D U ÇÃ O}

No Brasil, a regulamentação da profissão de nutricionista ocorreu em 24 de abril de 1967, quando foi sancionada pelo então Presidente da República, General Artur da Costa e Silva, a Lei $n^{\circ} 5.276$, que dispõe sobre a profissão de nutricionista, regula seu exercício e dá outras providências. Esse instrumento legal vigorou até 17 de setembro de 1991, quando foi revogado pela Lei $\mathrm{n}^{\circ} 8.234$, atualmente em vigor ${ }^{1-3}$.

Entretanto, a trajetória de organização, mobilização e luta pela regulamentação e legitimidade da profissão de nutricionista no País completou, em 2009, 70 anos de história. Essa trajetória teve seu início no final da década de 1930 e primeiros anos da década de 1940, em pleno decorrer do chamado Estado Novo (1937-1945), liderado pelo Presidente Getúlio Vargas. Nesse contexto histórico, identificado como um momento de transição político-econômica e social que propiciou as bases para a consolidação da sociedade capitalista urbano-industrial no país, foram criados, em 1939, os cursos técnicos de nível médio para formação de nutricionistas-dietistas, embriões dos atuais cursos de graduação em nutrição ${ }^{1-9}$.

O reconhecimento da profissão como de nível superior só ocorreu vinte e três anos depois da criação dos dois primeiros cursos técnicos de nível médio, quando o então Conselho Federal de Educação (CFE), órgão do Ministério da Educação, emitiu o Parecer n 265, de 19 de outubro de 1962. O referido parecer reconhecia os cursos de nutricionistas como de nível superior, estabelecia o primeiro currículo mínimo e fixava a duração de três anos para a formação de nutricionistas no país ${ }^{1-3}$.

Na luta pelo reconhecimento da profissão como de nível superior, vale destacar o importante papel desempenhado pelos seis cursos até então existentes e pela Associação Brasileira de Nutricionistas (ABN). A ABN, fundada em 31 de agosto 
de 1949, sediada no Rio de Janeiro, além de seu caráter técnico-científico e cultural, voltado ao desenvolvimento de estudos e pesquisas no campo da nutrição, passou a ser a primeira entidade brasileira a representar e a defender os interesses dos nutricionistas/dietistas. Em decorrência, no Brasil, em 31 de agosto, comemora-se o dia do nutricionista ${ }^{1-3,10}$.

Na década de 1970, dois importantes fatos históricos relacionados à profissionalização do nutricionista brasileiro merecem ser destacados. $\mathrm{O}$ primeiro diz respeito à fixação, pelo CFE, em 1974, do segundo currículo mínimo dos cursos de nutrição, que estabelecia uma carga horária total de 2880 horas a ser integralizada em quatro anos. O segundo é a aprovação da Lei $n^{\circ} 6.583$, de 20 de outubro de 1978, que cria os Conselhos Federal e Regionais de Nutricionistas, regula seu funcionamento, e dá outras providências. Com a aprovação da referida lei, os nutricionistas brasileiros passam a dispor de um órgão específico com a finalidade de fiscalizar o exercício da profissão, organizar, disciplinar e desenvolver a categoria e lutar pelos seus interesses. Ao final dos anos 1970, também teve início o processo de criação das associações profissionais (ou pré-sindicais), que deram origem aos Sindicatos de Nutricionistas em vários estados brasileiros, entidades responsáveis a partir de então pela defesa das questões trabalhistas relacionadas ao mercado de trabalho desses profissionais (salário, jornada e demais condições de trabalho) $)^{1-4,9,11,12}$.

O presente artigo tem como objetivo realizar uma análise histórica dos 70 anos da profissão de nutricionista no Brasil, procurando analisar a expansão do número de cursos de graduação em nutrição e de nutricionistas no país e discutir a identidade atual do nutricionista brasileiro, a partir de variáveis socioeconômicas e demográficas e áreas de atuação profissional. A base empírica do estudo foi constituída pelas estatísticas do número de profissionais e de cursos de graduação em nutrição disponibilizadas pelo Sistema Conselhos Federal e Regionais de Nutricionistas (CFN/CRNs) ${ }^{13,14}$ e pelos dados da pesquisa "Inserção Profissional dos Nutricionistas no Brasil", realizada em 2005, pelo CFN ${ }^{15}$.

\section{A E V O LU ÇÃ O DO NÚ M ERO DE CURSOS DE GRADUAÇÃO E M NUTRIÇÃO NO BRASIL (1939 - a g o s t o/2009)}

Estudos anteriores apontam que o processo histórico de evolução da profissão de nutricionista no Brasil teve seu início em outubro de 1939, quando foi criado o atual curso de graduação em nutrição do Departamento de Nutrição da Faculdade de Saúde Pública da Universidade de São Paulo (USP). Na década de 1940, foram criados mais três cursos para formação de nutricionistas: os atuais curso de graduação em nutrição da Universidade do Rio de Janeiro (UNI-RIO) (1940), curso de graduação em nutrição da Universidade Estadual do Rio de Janeiro (UERJ) (1944) e curso de graduação em nutrição do Instituto de Nutrição Josué de Castro da Universidade Federal do Rio de Janeiro (UFRJ) (1948) ${ }^{1-11}$. No entanto, vale ressaltar que, nos últimos dias, informações disponíveis na Internet põem em discussão a questão da primazia da instituição que desencadeou tal processo. Fontes documentais reunidas no Centro Estadual de Educação Tecnológica Paula Souza atestam que o processo histórico de evolução da profissão de nutricionista no Brasil teve seu início em março de 1939, com a criação dos Cursos Técnicos em Nutrição e Dietética na Superintendência do Ensino Profissional do Estado de São Paulo (http://www.cpscetec.com.br/memorias 170_anos/historico.html). Por outro lado, informações disponíveis na página eletrônica da UNI-RIO atestam que essa instituição teve o privilégio de ser pioneira no País em matéria de alimentação com base científica, a partir da criação, em 1939, do Curso Técnico de Auxiliares de Alimentação da Escola Central de Nutrição do então Estado da Guanabara, embrião do posterior curso para formação de nutricionistas (http://www.unirio.br/ ccbs/nutricao/nutricao.htm).

Nas décadas de 1950 e 1960, foram criados mais três cursos para formação de nutricionistas, 
os atuais curso de graduação em nutrição da Escola de Nutrição da Universidade Federal da Bahia (UFBA) (1956), curso de graduação em nutrição do Departamento de Nutrição da Universidade Federal de Pernambuco (UFPE) (1957) e curso de graduação em nutrição da Universidade Federal Fluminense (UFF) (1968) 1-3,7,9,11.

Assim, ao final da década de 1960 e início de 1970, existiam sete cursos para formação de nutricionistas no Brasil, todos vinculados a instituições públicas, ofertando cerca de 570 vagas anuais $2,4,11,16$ (Tabela 1).

Na década de 1970, sobretudo a partir de 1976, com a instituição do II Programa Nacional de Alimentação e Nutrição (II PRONAN), desenvolvido sob a coordenação do então Instituto Nacional de Alimentação e Nutrição (INAN), autarquia vinculada ao Ministério da Saúde, verificou-se um acelerado processo de criação de novos cursos para formação de nutricionistas no Brasil. Nesse sentido, uma vez que uma das diretrizes do II PRONAN consistia em estimular o processo de formação e capacitação de recursos humanos em nutrição, no período de 1970 a 1981, expandiu-se de 7 para 30 (21 públicos e 9 privados) o número de cursos de graduação em nutrição existentes no país, o que representou um aumento de $428,6 \%$. Nesse período, a oferta de vagas nesses cursos passou de 570 para 1 592, significando um aumento de 279,3\% (Tabela 1). A partir desse período, teve início o processo de criação dos cursos do setor privado, os quais em 1980 cor- respondiam a 30\% do total existente e eram responsáveis por $48 \%$ do total de vagas oferecidas no país $2,4,11,16$.

No período entre 1981 e 1996, o número de cursos passou de 30 para 45 (22 públicos e 23 privados), representando uma expansão de $642,9 \%$ em relação ao ano de 1970 , sendo esse aumento quase que exclusivamente determinado pela criação de 14 novos cursos privados. Nesse período, a oferta de vagas passou de 1592 para 3 643: um aumento de 639,1\% em relação a 19702,16 (Tabela 1).

A partir de 1996, com a instituição da Lei de Diretrizes e Bases da Educação (LDB), o processo de ampliação do número de cursos tem sido assustador. Essa expansão tem sido concentrada quase exclusivamente no setor privado, muito embora a partir de 2003 tenha ocorrido uma ligeira expansão do número de cursos e vagas nas instituições públicas de ensino superior. Até 31 de dezembro de 1996 existiam em todo o Brasil 45 cursos de graduação em nutrição. De janeiro de 1997 a agosto de 2009 surgiram mais 346 novos cursos de nutrição no Brasil. Portanto, do total de cursos existentes em agosto de 2009, 67 são públicos - federal, estadual ou municipal - e 324 são privados, ofertando um total de 49185 vagas anuais 2,13,16-18.

Em relação à distribuição geográfica, dos 391 cursos de graduação em nutrição existentes em agosto de 2009, 221 (56,5\%) localizam-se na Região Sudeste; 64 (16,4\%) na Região Sul; 58

Tabela 1. Distribuição do número de cursos e de vagas nos cursos de graduação em nutrição no Brasil nos anos de 1970, 1981, 1996, 01/2000, 01/2003, 08/2005, 04/2008 e 08/2009.

\begin{tabular}{|c|c|c|c|c|c|c|}
\hline \multirow{2}{*}{ Mês/Ano } & \multicolumn{4}{|c|}{ Cursos de graduação em nutrição } & \multicolumn{2}{|c|}{ Número de vagas } \\
\hline & Públicos & Privados & Total & Aumento \% & Número & Aumento \% \\
\hline 1970 & 7 & - & 7 & 100,0 & 570 & 100,0 \\
\hline 1981 & 21 & 9 & 30 & 428,6 & 1592 & 279,3 \\
\hline 1996 & 22 & 23 & 45 & 642,9 & 3643 & 639,1 \\
\hline $1 / 2000$ & 22 & 84 & 106 & 1514,3 & 8000 & 1403,5 \\
\hline $1 / 2003$ & 32 & 137 & 169 & 2414,3 & 15488 & 2717,2 \\
\hline $8 / 2005$ & 32 & 227 & 259 & 3700,0 & 24067 & 4222,3 \\
\hline $4 / 2008$ & 50 & 294 & 344 & 4914,0 & 43328 & 7601,4 \\
\hline $8 / 2009$ & 67 & 324 & 391 & 5585,7 & 49185 & 8628,9 \\
\hline
\end{tabular}

Fonte: Elaborada a partir dos dados de Bosi ${ }^{4}$ e Calado ${ }^{13,16-18}$. 
(14,8\%) na Região Nordeste; 30 (7,7\%) na Região Centro-Oeste e 18 (4,6\%) na Região Norte ${ }^{13}$.

Os 391 cursos de graduação em nutrição existentes em agosto de 2009 distribuem-se por 26 unidades da federação, sendo as dez maiores concentrações verificadas nos estados de São Paulo (108 ou 27,6\%), Minas Gerais (71\% ou 18,2\%), Rio de Janeiro (33 ou 8,4\%), Paraná (30 ou 7,7\%), Rio Grande do Sul (21 ou 5,4\%), Bahia ( 19 ou 4,9\%), Goiás (13 ou 3,4\%), Santa Catarina (13 ou 3,4\%), Distrito Federal (9 ou 2,3\%) e Espírito Santo ( 9 ou 2,3\%). Apenas o estado de Roraima ainda não dispõe de curso de graduação em nutrição ${ }^{13}$ (Tabela 2).

A expansão dos cursos de nutrição pós-LDB deu-se em todas as regiões do país e ca-

Tabela 2. Distribuição do número absoluto e percentual de cursos de graduação em nutrição no Brasil, por unidade da federação, em agosto de 2009.

\begin{tabular}{|c|c|c|}
\hline Unidade da Federação & Total & $\%$ \\
\hline São Paulo & 108 & 27,6 \\
\hline Minas Gerais & 71 & 18,2 \\
\hline Rio de Janeiro & 33 & 8,4 \\
\hline Paraná & 30 & 7,7 \\
\hline Rio Grande do Sul & 21 & 5,4 \\
\hline Bahia & 19 & 4,9 \\
\hline Goiás & 13 & 3,4 \\
\hline Santa Catarina & 13 & 3,4 \\
\hline Distrito Federal & 9 & 2,3 \\
\hline Espírito Santo & 9 & 2,3 \\
\hline Pernambuco & 8 & 2,1 \\
\hline Amazonas & 7 & 1,8 \\
\hline Piauí & 7 & 1,8 \\
\hline Maranhão & 6 & 1,5 \\
\hline Paraíba & 5 & 1,3 \\
\hline Rio Grande do Norte & 5 & 1,3 \\
\hline Ceará & 5 & 1,3 \\
\hline Mato Grosso do Sul & 5 & 1,3 \\
\hline Pará & 4 & 1,0 \\
\hline Mato Grosso & 3 & 0,7 \\
\hline Rondônia & 3 & 0,7 \\
\hline Alagoas & 2 & 0,5 \\
\hline Amapá & 2 & 0,5 \\
\hline Acre & 1 & 0,2 \\
\hline Sergipe & 1 & 0,2 \\
\hline Tocantins & 1 & 0,2 \\
\hline Total & 391 & 100,0 \\
\hline
\end{tabular}

Fonte: Elaborada a partir dos dados de Calado ${ }^{13}$. racterizou um processo de interiorização (aumento do número de cursos em cidades do interior), sobretudo a partir de 2003. Na Região Norte, o primeiro curso de nutrição foi criado em 1973, e o segundo em 1999, levando portanto 26 anos para que começassem a surgir novos cursos. Na Região Centro-Oeste, a criação do primeiro curso data de 1975; até 1996 existiam 4 cursos, e em agosto de 2009, 30 cursos. Na Região Nordeste, entre 1976 e 1986, ocorreu a criação de mais seis cursos nos estados da Paraíba (1976), Piauí (1976), Rio Grande do Norte (1976), Ceará (1977), Alagoas (1978) e Bahia (1986), de modo que até 1996 existiam 8 cursos, e em agosto de 2009 são encontrados 58 cursos em todos os estados da região. Na Região Sudeste, até 1996 existiam 23 cursos, e em agosto de 2009, 221. Na Região Sul, o primeiro curso foi criado em 1972, na Universidade do Vale do Rio dos Sinos (Unisinos), em São Leopoldo, sendo o primeiro curso privado do país; até 1996 existiam 9 cursos, e em agosto de 2009 somam 64 cursos $^{13}$.

Em relação ao processo de interiorização dos cursos de nutrição verificado a partir de 2003, chama atenção a criação em cidades com menos de 50 mil habitantes (dados publicados pelo IBGE referentes à estimativa de população em $1^{\circ}$ de julho de 2009) ${ }^{19}$. Vale destacar a criação dos cursos em Goiás, na cidade de Mineiros (48 329 habitantes); na Paraíba, na cidade de Cuité (20 834 habitantes); no Rio Grande do Norte, na cidade de Santa Cruz (35 095 habitantes); no Espírito Santo, nas cidades de Alegre (31 143 habitantes) e Santa Teresa (20 742 habitantes); em Minas Gerais, nas cidades de Além Paraíba (34 591 habitantes), Bom Despacho (44 265 habitantes), Diamantina (46 372 habitantes), Machado (39 509 habitantes), Nanuque (41 329 habitantes), Ouro Fino (32 639 habitantes) e São Lourenço (42 688 habitantes); em São Paulo, nas cidades de Adamantina (34 424 habitantes), Jaguariúna (41 107 habitantes) e Santa Fé do Sul (29 192 habitantes), e em Santa Catarina, na cidade de Videira (46 585 habitantes) ${ }^{13,19}$. 
Outra característica é o aumento de cursos em instituições privadas, observando-se, entretanto, em menor proporção e, sobretudo, nos últimos anos, um crescimento dos cursos em instituições públicas, principalmente federais. No Brasil, até 1996, existiam 22 cursos de nutrição em instituições públicas de ensino superior - federal, estadual ou municipal -, após 1996, foram criados mais 45 cursos em instituições públicas, sendo 3 na Região Norte, 3 na Região Centro-Oeste, 10 na Região Nordeste, 21 na Região Sudeste e 8 na Região Sul13.

Torna-se interessante realizar uma comparação desses dados com aqueles dos demais cursos de graduação da área da saúde e outros cursos de nível superior no País. Segundo informações do Instituto Nacional de Estudos e Pesquisas Educacionais Anísio Teixeira (INEP) ${ }^{20}$, em agosto de 2009, observava-se a seguinte distribuição do número de cursos de nível superior da área de saúde, incluindo educação física, em ordem decrescente: 1) educação física, com 897 cursos, sendo $23 \%$ ( $n=207$ ) públicos; 2 ) enfermagem, com 791 cursos, sendo 18,8\% ( $n=149)$ públicos; 3) farmácia, com 534 cursos, sendo $22,1 \%$ ( $n=118$ ) públicos; 4) fisioterapia, com 520 cursos, sendo 9\% ( $n=47)$ públicos; 5$)$ nutrição, com 391 cursos, sendo $17,1 \%$ ( $n=67)$ públicos; 6 ) odontologia, com 209 cursos, sendo 26,3\% (n=55) públicos e 7) medicina, com 177 cursos, sendo 37,9\% ( $\mathrm{n=67)}$ públicos.

\section{A EXPANSÃ O DO NÚ MERO DE NUTRICIONISTAS NO BRASIL (1989 - j u n h o/2009)}

Consequentemente ao acelerado aumento do número de cursos e de vagas, nos últimos vinte anos também se observou uma ampliação quantitativa substancial de nutricionistas no País. Conforme estatísticas do Conselho Federal de Nutricionistas (CFN), em 1989, existia um contingente de 11898 nutricionistas no Brasil, perfazendo uma relação de cerca de um nutricionista para cada 11500 habitantes (Tabela 3). Com base nas estatísticas do CFN, até 30 de junho de 2009, existia um efetivo de 60554 nutricionistas registrados nos dez conselhos regionais existentes no País. Portanto, em junho de 2009, diante do efetivo de nutricionistas existentes, verificou-se uma relação de cerca de um nutricionista para cada 3164 habitantes. Entre 2000 e 2008, observou-se uma duplicação do número de nutricionistas, passando de 28983 para 56217 profissionais no Brasil. Entre 2003 e 2008, a taxa de crescimento anual do número de nutricionistas variou de 1082 a 7709 novos nutricionistas/ano, perfazendo uma taxa média de crescimento anual da profissão de 4041 novos nutricionistas/ano 14,19,21.

Portanto, com base em estudo anterior, entre 1989 e 2007, observou-se um aumento de $421 \%$ no número de nutricionistas no Brasil21 .

Tabela 3. Distribuição do número total de nutricionistas, taxa de crescimento absoluto anual e da relação de nutricionistas por habitante no Brasil no período de 1989 a junho de 2009.

\begin{tabular}{lccc}
\hline Ano & Total de nutricionistas & $\begin{array}{c}\text { Crescimento absoluto do número de } \\
\text { nutricionistas entre os anos }\end{array}$ & Relação nutricionistas/habitantes \\
\hline 1989 & 11898 & - & $1 / 11500$ \\
2000 & 28983 & 17085 & $1 / 5559$ \\
2001 & 31971 & 2988 & $1 / 5391$ \\
2003 & 33328 & 1357 & $1 / 5220$ \\
2004 & 34410 & 1082 & $1 / 5050$ \\
2005 & 37238 & 2828 & $1 / 4952$ \\
2006 & 44947 & 7709 & $1 / 4155$ \\
2007 & 50041 & 5094 & $1 / 3757$ \\
2008 & 56217 & 6176 & $1 / 3373$ \\
$30 / 06 / 2009$ & 60554 & 4337 & $1 / 3162$ \\
\hline
\end{tabular}

Fonte: Elaborada a partir das estatísticas do número de nutricionistas inscritos no Conselho Federal de Nutricionistas (CFN) (http://www.cfn.org.br) e dados populacionais do Instituto Brasileiro de Geografia e Estadística (IBGE) (http://www.ibge.gov.br/home). 
Entre 1989 e junho de 2009, a taxa de aumento do número de nutricionistas no Brasil passou a ser de $509 \%$.

Em relação à distribuição dos 60554 nutricionistas existentes em junho de 2009, por regiões, verifica-se que, desse total, $32516(53,7 \%)$ localizam-se na Região Sudeste; 11075 (18,3\%) na Região Sul; 8357 (13,8\%) na Região Nordeste; 4815 (7,9\%) na Região Centro-Oeste e 3791 $(6,3 \%)$ na Região Norte ${ }^{14}$. Os dez estados brasileiros onde se verificavam as maiores concentrações de nutricionistas, em ordem decrescente, eram: São Paulo, com 17254 (28,5\%); Rio de Janeiro, com 8559 (14,1\%); Minas Gerais, com 5523 (9,1\%); Rio Grande do Sul, com 5079 (8,4\%); Paraná, com 4143 (6,8\%); Bahia, com 2393 (4,0\%); Distrito Federal, com 2152 (3,6\%); Santa Catarina, com 1853 (3,1\%); Pará, com 1447 (2,4\%), e Pernambuco, com 1423 (2,3\%) (Tabela 4).

Torna-se relevante discutir esses dados à luz das estatísticas fornecidas pelas demais profissões da área da saúde. De acordo com o Conselho Federal de Medicina (CFM) ${ }^{22}$, em agosto de 2009, o total de médicos inscritos no Brasil era de 510 397, enquanto o total de médicos ativos era de 343 048. Com base nesses números do CFM, estima-se uma relação de cerca de um médico inscrito para cada 376 habitantes e de cerca de um médico ativo para cada 559 habitantes no País ${ }^{22}$. De acordo com o Conselho Federal de Odontologia (CFO), em 11 de agosto de 2009, o total de cirurgiões-dentistas ${ }^{23}$ inscritos no Brasil era de 227 633, estimando-se uma relação de cerca de um cirurgião-dentista inscrito para cada 842 habitantes ${ }^{23}$. De acordo com o Conselho Federal de Farmácia (CFF), em dezembro de 2008, o total de farmacêuticos inscritos no Brasil ${ }^{24}$ era de 122 915, estimando-se uma relação de cerca de um farmacêutico inscrito para cada 1559 habitantes $^{24}$. De acordo com dados do Ministério da Saúde (MS), em 2006, o total de enfermeiros ${ }^{25}$ no Brasil era de 102 359, estimando-se uma relação de cerca de um enfermeiro para cada 1833 habitantes ${ }^{25}$.
A partir das estatísticas das cinco profissões de saúde analisadas (medicina, enfermagem, odontologia, farmácia e nutrição), a nutrição é, portanto, a que apresenta o menor contingente de profissionais. Embora alguns autores ${ }^{26,27}$ ainda façam referência ao parâmetro de um médico para cada mil habitantes, atribuído à Organização Mundial da Saúde (OMS), um documento elaborado em 2003 pela Organização Pan-Americana da Saúde (OPAS) e OMS ${ }^{28}$ expressa que essas instituições não recomendam e nem estabelecem taxas ideais ou desejáveis do número de médicos ou outros profissionais da saúde por habitante. De acordo com esse documento, a definição de índices, como número de médicos por habitantes, depende de fatores regionais, socioeconômicos,

Tabela 4. Distribuição do número de nutricionistas no Brasil e por unidade da federação, até 30 de junho de 2009.

\begin{tabular}{|c|c|c|}
\hline Unidade da Federação & Total & $\%$ \\
\hline São Paulo & 17254 & 28,5 \\
\hline Rio de Janeiro & 8559 & 14,1 \\
\hline Minas Gerais & 5523 & 9,1 \\
\hline Rio Grande do Sul & 5079 & 8,4 \\
\hline Paraná & 4143 & 6,8 \\
\hline Bahia & 2393 & 4,0 \\
\hline Distrito Federal & 2152 & 3,6 \\
\hline Santa Catarina & 1853 & 3,1 \\
\hline Pará & 1447 & 2,4 \\
\hline Pernambuco & 1423 & 2,3 \\
\hline Amazonas & 1192 & 2,0 \\
\hline Goiás & 1077 & 1,8 \\
\hline Espírito Santo & 1044 & 1,7 \\
\hline Ceará & 921 & 1,5 \\
\hline Rio Grande do Norte & 830 & 1,4 \\
\hline Paraíba & 809 & 1,3 \\
\hline Mato Grosso & 737 & 1,2 \\
\hline Mato Grosso do Sul & 677 & 1,1 \\
\hline Rondônia & 648 & 1,1 \\
\hline Alagoas & 663 & 1,1 \\
\hline Piauí & 619 & 1,0 \\
\hline Maranhão & 394 & 0,7 \\
\hline Amapá & 329 & 0,5 \\
\hline Sergipe & 135 & 0,2 \\
\hline Tocantins & 119 & 0,2 \\
\hline Acre & 96 & 0,2 \\
\hline Roraima & 79 & 0,1 \\
\hline Outros & 359 & 0,6 \\
\hline Total & 60554 & 100,0 \\
\hline
\end{tabular}

Fonte: Elaborada a partir dos dados do CFN ${ }^{14}$. 
culturais e epidemiológicos, entre outros, que diferem de região para região, país para país. Sendo assim, o estabelecimento de um valor ideal a ser aplicado de maneira generalizada por todos os países teria pouca validade/utilidade ${ }^{28}$.

\section{A ATUAL IDENTIDADE DO NUTRICIONISTA B R A S I LE I R O}

De acordo com pesquisa do Conselho Federal de Nutricionistas ${ }^{15}$, realizada em 2005 com um total de 2492 nutricionistas, a "identidade" profissional do nutricionista brasileiro com base nas variáveis sexo, faixa etária, estado civil, cor da pele, área geográfica de atuação e renda média mensal apresentava o seguinte perfil (Tabela 5): $96,5 \%$ pertenciam ao sexo feminino; $79,4 \%$ tinham entre 20 e 40 anos; 53,6\% eram solteiros; 79,1\% de cor branca; 66,7\% com atuação profissional nas capitais dos estados brasileiros e com renda média mensal de $\mathrm{R} \$ 1.616,00$. Os entrevistados da referida pesquisa foram estratificados proporcionalmente pelo efetivo de profissionais inscritos nos distintos Conselhos Regionais de Nutricionistas (CRN), usando-se 95,0\% de intervalo de confiança com margem de erro de 1,89 pontos percentuais $^{15}$.

Em relação à variável sexo, os dados denotam uma profissão predominantemente feminina, característica inerente às origens históricas desse profissional no Brasil e que continua se reproduzindo nos dias atuais ${ }^{29-32}$. Alguns estudos têm procurado associar essa característica da identidade do nutricionista brasileiro às questões que dizem respeito ao gênero, à participação da mulher no mercado de trabalho e ao seu papel na sociedade brasileira. Essas questões não são exclusivas da profissão de nutricionista, mas também são evidenciadas em outras profissões com predominância feminina, como é o caso da enfermagem, serviço social, letras e pedagogia ${ }^{29-32}$.

Em relação à faixa etária, o perfil é de uma profissão com maior densidade de adultos jovens: 52,6\% têm entre 20 e 30 anos de idade. Esse perfil parece estar intimamente relacionado ao processo de expansão do número de cursos e vagas verificado no período pós-LDB ${ }^{16}$, uma vez que a pesquisa também identificou que $50 \%$ dos

Tabela 5. Distribuição dos nutricionistas brasileiros ( $n=2$ 492) segundo as variáveis sexo, faixa etária, estado civil, cor da pele/etnia, área geográfica de atuação, áreas de atuação profissional e renda média mensal por área de atuação. Brasil, 2005

\begin{tabular}{|c|c|}
\hline Variáveis & $\%$ \\
\hline \multicolumn{2}{|l|}{ Sexo } \\
\hline Feminino & 96,5 \\
\hline Masculino & 3,5 \\
\hline \multicolumn{2}{|l|}{ Faixa etária (anos) } \\
\hline 20 a 30 & 52,6 \\
\hline 31 a 40 & 26,8 \\
\hline 41 a 50 & 16,8 \\
\hline 51 a 60 & 3,3 \\
\hline$>60$ & 0,5 \\
\hline \multicolumn{2}{|l|}{ Estado civil } \\
\hline Solteiro & 53,6 \\
\hline Casado & 39,6 \\
\hline Outros & 6,8 \\
\hline \multicolumn{2}{|l|}{ Cor da pele/etnia } \\
\hline Branca & 79,1 \\
\hline Parda & 13,7 \\
\hline Amarela & 3,5 \\
\hline Preta & 3,4 \\
\hline Indígena & 0,3 \\
\hline \multicolumn{2}{|l|}{ Área geográfica de atuação } \\
\hline Capital & 66,7 \\
\hline Interior & 30,5 \\
\hline Ambos & 2,8 \\
\hline \multicolumn{2}{|l|}{ Área de atuação profissional } \\
\hline Nutrição clínica & 41,7 \\
\hline Alimentação coletiva & 32,3 \\
\hline Ensino (docência) & 9,4 \\
\hline Saúde coletiva & 8,8 \\
\hline Nutrição esportiva & 4,1 \\
\hline Indústria de alimentos & 3,7 \\
\hline Renda média mensal por área de atuação profissional & Reais \\
\hline Ensino (docência) & 1884,35 \\
\hline Indústria de alimentos & 1525,89 \\
\hline Nutrição clínica & 1479,82 \\
\hline Saúde coletiva & 1475,56 \\
\hline Alimentação coletiva & 1392,21 \\
\hline Nutrição esportiva & 1276,06 \\
\hline Renda média nacional & 1616,00 \\
\hline
\end{tabular}

Fonte: Elaborada a partir dos dados do $\mathrm{CFN}^{15}$. 
profissionais entrevistados tinham até cinco anos de formados ${ }^{15}$.

O fato de o estado civil solteiro representar 53,6\% dos nutricionistas brasileiros em 2005 pode estar relacionado com a faixa etária predominante identificada na pesquisa (20 a 30 anos de idade) ${ }^{15}$, hipótese que precisa ser mais bem analisada em futuras investigações.

O fato de a maior densidade dos nutricionistas entrevistados declararem-se da cor branca ${ }^{15}$ pode ser indicativo de um possível viés seletivo de raça/etnia e/ou de classe social existente no processo de profissionalização do nutricionista e de outras profissões de nível superior no país, hipótese que também precisa ser mais bem investigada. Estudo realizado sobre a inserção profissional dos médicos brasileiros observou que no ano de 2003 a proporção de médicos da cor/raça branca era de $88 \%$, sendo, portanto, muito mais elevada do que a proporção de brancos na população brasileira em geral, estimada pela Pesquisa Nacional por Amostra de Domicílios (PNAD) de 2003 em 53\%33. É possível que nos próximos anos, como consequência da chamada política afirmativa introduzida no processo seletivo do ensino superior do país a partir de $1996^{34}$, esse perfil de raça/etnia e/ou de classe social seja mais igualitário.

A concentração da maioria dos nutricionistas nas capitais do país denota um perfil de desigualdade na distribuição geográfica desses profissionais. A pesquisa revelou que as capitais e os centros urbanos exercem maior atração para fixação dos profissionais e isso talvez se explique não apenas pela existência de um mercado de trabalho mais amplo e com melhores condições, mas pela oferta de outras condições de vida não existentes nas cidades do interior. Vale destacar que esse perfil não é específico para a profissão de nutricionista, uma vez que outras profissões de nível superior também apresentam perfis de distribuição geográfica com maior densidade de concentração nas capitais, centros urbanos e regiões geográficas mais desenvolvidas e com maior produto interno bruto (PIB) per capita, como é o caso da medicina ${ }^{26}$.

A renda média mensal é bem inferior às reivindicações salariais pleiteadas pelo movimento da categoria profissional a partir de meados dos anos $1980^{31}$. Àquela época, com base nas deliberações do I Encontro Nacional das Entidades de Nutricionistas, propunha-se um piso salarial de 10 salários-mínimos para uma jornada de trabalho de 30 horas semanais ${ }^{35}$. Observa-se, portanto, com base no valor do salário-mínimo vigente em 2005 ( $R \$ 300,00)$, ano de realização da pesquisa, que a renda média mensal dos nutricionistas brasileiros era equivalente a apenas 5,4 salários-mínimos.

Conforme as seis grandes áreas de atuação profissional investigadas pelo CFN, o perfil da "identidade" profissional do nutricionista assumia a seguinte distribuição: $41,7 \%$ atuando em nutrição clínica, 32,2\% em alimentação coletiva, 9,4\% em ensino (docência), 8,8\% em saúde coletiva, $4,1 \%$ em nutrição esportiva e 3,7\% em indústria de alimentos ${ }^{15}$ (Tabela 5).

Em relação às áreas de atuação profissional, nas últimas décadas, têm sido observadas intensas diversificação e ampliação, fato que pode estar associado ao processo de grande elevação do número de cursos e profissionais no Brasil. Atualmente, o Conselho Federal de Nutricionistas reconhece a existência de sete grandes áreas de atuação profissional do nutricionista: nutrição clínica, alimentação coletiva, saúde coletiva, ensino (docência), nutrição esportiva, indústria de alimentos e marketing em alimentação e nutrição ${ }^{36}$. Entretanto, observa-se que dentro de cada uma dessas grandes áreas de atuação profissional está ocorrendo um amplo processo de divisão/especialização dos seus objetos específicos de estudo e trabalho. Nesse aspecto, é preciso mapear esses novos campos de atuação/especialização do trabalho do nutricionista no Brasil, procurando realizar investigações que atualizem os dados da pesquisa do CFN de $2005^{15}$.

Dentro do crescente processo de divisão/especialização do trabalho do nutricionista no 
Brasil, verificado nas últimas décadas, na área de nutrição clínica, que à época concentrava a maior densidade de nutricionistas, observam-se subáreas de atuação por patologias, grupos etários ou outras especializações, tais como a atuação do nutricionista em oncologia, nefrologia, cardiologia, diabetes, transtornos alimentares, obesidade, pediatria, geriatria, personal diet, consultórios e clínicas, hospitalização domiciliar etc. 15,36-39.

$\mathrm{Na}$ área de alimentação coletiva, que à época concentrava a segunda maior densidade de nutricionistas do País, observa-se a expansão da atuação para setores específicos como restaurantes comerciais, fast-food e similares, assessoria em supermercados e padarias, hotelaria, SPA, controle de qualidade e vigilância sanitária etc. ${ }^{15,} 30,36,37,40-42$.

A nutrição em saúde coletiva, por sua vez, à época ocupando o quarto lugar na concentração do número de nutricionistas no País, consolida e amplia a área de atuação desse profissional no campo das políticas públicas, tais como no Programa Nacional de Alimentação Escolar (PNAE), no Programa de Alimentação do Trabalhador (PAT), no Programa Bolsa Família, na Estratégia Saúde da Família, a partir da criação dos Núcleos de Atenção à Saúde da Família (NASF) etc. ${ }^{15,36-37,43-46}$.

De forma semelhante, a recente área de atuação profissional do nutricionista - nutrição esportiva -, ocupa os espaços das academias e de distintas modalidades esportivas no país (futebol, vôlei, natação, atletismo etc. $)^{15,36-37,47}$.

Por fim, a pesquisa realizada pelo CFN também identificou que $47,4 \%$ dos nutricionistas brasileiros tinham cursado ou estavam realizando cursos de especialização, 9,4\% tinham ou estavam cursando mestrado e apenas 2,4\% tinham ou estavam cursando doutorado ${ }^{15}$. Esses dados apontam para a necessidade de investimentos no processo de formação continuada do nutricionista no que se refere à ampliação das possibilidades de realização de cursos de pós-graduação estrito senso (mestrado e doutorado).

\section{CONSIDERAÇÕES FINAIS}

Este estudo sobre a trajetória histórica da profissão de nutricionista no Brasil permitiu demonstrar que, ao longo dos 70 anos investigados, houve um expressivo aumento na quantidade de cursos e de profissionais no País. A instituição da Lei de Diretrizes e Bases da Educação Brasileira (Lei n 9.394, de 20 de dezembro de 1996) constituiu o elemento catalisador desse processo de expansão acelerada do número de cursos e nutricionistas brasileiros. Assim, entre 1939 e 1996 (57 anos) foram criados 45 cursos de nutrição, enquanto entre 1997 e agosto de 2009 (12 anos) foram criados 346. Simultaneamente, entre 2000 e junho de 2009, observou-se uma duplicação do efetivo de nutricionistas, passando de 28983 para 60544 o número de profissionais no País. Portanto, se for mantida a taxa de reprodução ou crescimento médio anual verificada entre 2005 e 2008 (6 326 nutricionistas/ano), a tendência é uma nova duplicação do número atual de nutricionistas nos próximos dez anos.

A Região Sudeste e o Estado de São Paulo continuam com as maiores concentrações de cur$\operatorname{sos}(56,5 \%$ e $27,6 \%$, respectivamente) e de nutricionistas $(53,7 \%$ e $28,5 \%$, respectivamente) do País. Entretanto, observou-se que no pós-LDB a expansão no número de cursos e de profissionais se deu em todas as regiões, ressaltando-se o processo de interiorização e revitalização do setor público de ensino superior ocorrido a partir de 2003.

Os resultados também indicam uma sensível redução da relação nutricionista por habitante no Brasil: 3,6 vezes menor que a verificada em 1989. Entretanto, quando relacionada às cinco profissões de saúde analisadas (medicina, enfermagem, odontologia, farmácia e nutrição), observa-se que, em 2009, a nutrição é aquela que apresenta o menor contingente de profissionais e, consequentemente, a maior taxa de profissionais/ habitante.

Com base nos dados da pesquisa realizada pelo Conselho Federal de Nutricionistas, em 2005, 
o perfil do nutricionista brasileiro pode ser assim sintetizado: mulher, 20 a 30 anos de idade, branca, com predomínio de atuação em nutrição clínica em São Paulo (capital), cursando especialização em terapia nutricional em oncologia pediátrica, com uma renda mensal de $\mathrm{R} \$ 1.616,00$.

É provável que nos próximos cinco anos, em função de novos cursos de nutrição e do consequente lançamento de um maior número de profissionais no mercado de trabalho, o quadro ora traçado apresente nova configuração. Possivelmente ocorrerá uma redistribuição territorial dos nutricionistas, bem como modificações no mercado de trabalho e áreas de atuação profissional. Nesse sentido, investigações que possibilitem o monitoramento dessas possíveis alterações tornam-se cada vez mais imprescindíveis.

Em síntese, ao longo dos 70 anos de história do nutricionista no Brasil, ocorreu um expressivo aumento no número de cursos e de profissionais, houve um aperfeiçoamento dos métodos e dos instrumentos de trabalho, redesenharam-se o mercado e as condições de trabalho desses profissionais, e também se verificaram profundas e substanciais alterações no padrão de consumo, nos hábitos alimentares e no estado nutricional da população brasileira (não discutidas no presente artigo). Resta-nos indagar se essa ampliação quantitativa possibilitará um avanço qualitativo da atuação do nutricionista no Brasil.

Tomando como guia de ação as atuais Diretrizes Curriculares Nacionais do Curso de Graduação em Nutrição, urge à nossa incorporação imediata a luta pela defesa de um perfil para o nutricionista brasileiro que o identifique como profissional "com formação generalista, humanista e crítica. Capacitado a atuar, visando à segurança alimentar e a atenção dietética, em todas as áreas do conhecimento em que alimentação e nutrição se apresentem fundamentais para a promoção, manutenção e recuperação da saúde e para a prevenção de doenças de indivíduos ou grupos populacionais, contribuindo para a melhoria da qualidade de vida, pautado em princípios éticos, com reflexão sobre a realidade econômica, política, social e cultural".

\section{REFERÊ NCIAS}

1. Associação Brasileira de Nutrição. Histórico do nutricionista no Brasil-1939 a 1989: coletânea de depoimentos e documentos. São Paulo: Atheneu; 1991.

2. Vasconcelos FAG. O nutricionista no Brasil: uma análise histórica. Rev Nutr. 2002; 15(2):127-38. doi: 10.1590/S1415-52732002000200001.

3. Conselho Federal de Nutricionistas. 40 anos de re gulamentação da profissão. [acesso 2009 ago.19]. Disponível em: <http://www.cfn.org.br/>.

4. Bosi MLM. A face oculta da nutrição: ciência e ideologia. Rio de Janeiro: UFRJ; 1988.

5. L'Abbate S. As Políticas de Alimentação e Nutrição no Brasil. I. Período de 1940 a 1964. Rev Nutr. 1988; 1(2):87-138.

6. Costa D. A importância do ensino da nutrição: o seu desenvolvimento no Brasil. Rev Bras Méd. 1953; 10(9):645-50.

7. Coêlho HAL. Formação do profissional nutricionista na América Latina e no Brasil, com ênfase em Pernambuco. Rev Aliment Nutr. 1983; 4(11):47-51.

8. Maurício HV. Evolução da nutrição e do seu ensino no Brasil. Arq Bras Nutr. 1964; 20(2):117-34.

9. Ypiranga L, Gil MF. Formação profissional do nutricionista: por que mudar? In: Cunha DTO, Ypiranga L, Gil MF, organizadores. Anais do $2^{\circ}$ Seminário Nacional sobre o Ensino de Nutrição. Goiânia: FEBRAN; 1989.

10. Vasconcelos FAG. Os Arquivos Brasileiros de Nutrição: uma revisão sobre produção científica em nutrição no Brasil (1944 a 1968). Cad Saúde Pública. 1999; 15(2):303-16.

11. Brasil. Ministério da Educação e Cultura Secretaria de Ensino Superior. Os Cursos de nutrição no Brasil: evolução, corpo docente e currículo. Brasília: ME; 1983. Série de Cadernos de Ciências da Saúde, 6.

12. Sindicato dos Nutricionistas do Rio Grande do Sul. Um pouco da História do SINURGS. [acesso 2009 ago.19]. Disponível em: <http://www.sinurgs.org. br/quem.php>.

13. Calado CLA. Relação de endereços dos cursos de nutrição no Brasil. 2009. [acesso 2009 ago. 15]. Disponível em: <http://www.cfn.org.br/novosite/ arquivos/ESCOLA-NUTRIÇAO-AGOSTO-2009. $p d f>$.

14. Conselho Federal de Nutricionistas. Quadro estatístico do $2^{\circ}$ trimestre/2009 (1/4/2009 a 30/6/2009). [acesso 2009 ago. 15]. Disponível em: <http:// www.cfn.org.br/novosite/arquivos/ESTATISTICO-2TRIMESTRE-2009.pdf>. 
15. Conselho Federal de Nutricionistas. Inserção profissional dos nutricionistas no Brasil. Brasília: CFN; 2006 [acesso 2009 ago.15]. Disponível em: <http:// www.cfn.org.br/novosite/pdf/pesquisa.pdf>.

16. Calado CLA. A expansão dos cursos de nutrição no Brasil e a nova Lei de Diretrizes e Bases LDB 2003. [acesso 2009 ago. 23]. Disponível em: <http:// www.cfn.org.br/novosite/pdf/expansao.pdf>.

17. Calado CLA. Relação de endereços dos cursos de nutrição no Brasil. 2005. [acesso 2009 ago. 23]. Disponível em: <http://www.cfn.org.br/novosite/ pdf/escolas/agosto05.pdf>.

18. Calado CLA. Relação de endereços dos cursos de nutrição no Brasil. 2008. [acesso 2009 ago. 23]. Disponível em: <http://www.cfn.org.br/ novosite/ arquivos/cursos_nutricao_abril_2008.doc>.

19. Instituto Brasileiro de Geografia e Estatística. Estimativa de população em $1^{\circ}$ de julho de 2009. [acesso 2009 ago. 27]. Disponível em: <www.ibge. gov.br>.

20. Instituto Nacional de Estudos e Pesquisas Educacionais Anísio Teixeira Educação Superior. Cursos e instituições. Cursos. [acesso 2009 ago. 27]. Disponível em: <www.educacaosuperior.inep. gov.br>

21. Vasconcelos FAG, Rossi CE, Costa M. Evolução histórica do nutricionista em Santa Catarina (19802003). Rev Ciênc Saúde. 2005; 24(1/2):28-35.

22. Conselho Federal de Medicina. Estatísticas. Total de inscritos e ativos no Brasil. [acesso 2009 ago. 27]. Disponível em: <http://www.portalmedico. org.br/novoportal/index5.asp>.

23. Conselho Federal de Odontologia. Total de profissionais por Conselho Regional de Odontologia. Pesquisa Total de Profissionais por CRO. CRO: todos em 11 de agosto de 2009. [acesso 2009 ago.16]. Disponível em: <http://www.cfo.org.br/busca_ dados/totais/tot_prof_cro.asp>.

24. Conselho Federal de Farmácia. Números da Farmácia no Brasil. Estatísticas de dezembro de 2008. [acesso 2009 ago. 16]. Disponível em: <http://www. cff.org.br/\#[ajax]pagina\&id=138>.

25. Brasil. Ministério da Saúde. Indicadores e dados básicos-Brasil-2007 (IDB-2007). Número de profissionais de saúde por habitante. Número de enfermeiros segundo Unidades da Federal. 2006. [acesso 2009 ago. 24]. Disponível em: <http:// tabnet.datasus.gov.br/cgi/tabcgi.exe?idb2007/ e01.def>

26. Povoa L, Andrade MG. Distribuição geográfica dos médicos no Brasil: uma análise a partir de um modelo de escolha locacional. Cad Saúde Pública; 2006; 22 (8):1555-64.
27. Brasil. Ministério da Saúde. Indicadores e dados básicos Brasil 2000. Indicadores de recursos. Número de profissionais de saúde por habitante. Ficha de qualificação. [acesso 2009 ago. 22]. Disponível em: <http://tabnet.datasus.gov.br/cgi/idb2000/ recur.htm>.

28. Organização Pan-Americana da Saúde. Leitos por habitante e médicos por habitante. 2003. [acesso 2009 ago. 22]. Disponível em: <http://www.opas. org.br/sistema/fotos/leitos.pdf>.

29. Rotemberg S, Prado S. Nutricionista: quem somos? Rev Nutr. 1991; 4(1/2):40-64.

30. Akutsu RC. Os nutricionistas brasileiros: perfil profissional e demográfico. Rev Nutr. 2008; 21(1): 7-19. doi: 10.1590/\$1415-52732008000100002.

31. Costa NMSC. Revisitando os estudos e eventos sobre a formação do nutricionista no Brasil. Rev Nutr. 1999; 12(1):5-19.doi: 10.1590/S1415-5273 1999000100001.

32. Andrade LP, Lima ES. A formação e a prática do nutricionista: o gênero nas entrelinhas. Nutrire: Rev Bras Alim Nutr. 2003; 26:109-26.

33. Luiz RR, Bahia L. Renda e inserção profissional dos médicos brasileiros após instituição do Sistema Único de Saúde. Rev Saúde Pública. 2009; 43(4): 689-98.

34. Moehlecke S. Ação afirmativa: história e debates no Brasil. Cad Pesq. 2002; 117:197-217.

35. Gambardella AMD, Ferreira CF, Frutuoso MFP. Situação profissional de egressos de um curso de nutrição. Rev Nutr. 2000; 13(1):37-40. doi: 10.15 90/S1415-52732000000100005.

36. Conselho Federal de Nutricionistas. Resolução CFN n 380/2005 Dispõe sobre a definição das áreas de atuação do nutricionista e suas atribuições, estabelece parâmetros numéricos de referência por área de atuação e dá outras providências. [acesso 2009 ago. 20]. Disponível em: <http://www.cfn. org.br/novosite/pdf/res/2005/res380.pdf>.

37. Conselho Federal de Nutricionistas. Profissão de nutricionista completa 40 anos de regulamentação. Revista do CFN [Internet]. 2007 [acesso 2009 ago. 22]; 5(21):8-9. Disponível em: http://www.cfn.org. $\mathrm{br} /$ novosite/pdf/revistas/21.pdf>.

38. Oliveira TRPR, Radicchi ALA. Inserção do nutricionista na equipe de atendimento ao paciente em reabilitação física e funcional. Rev Nutr. 2005; 18(5): 601-11. doi: 10.1590/\$1415-527320050005000 03.

39. Vieira MA, Lima IN, Petilik MEl. Abordagem ambulatorial do nutricionista em anemia hemolítica. Rev Nutr. 1999; 12(1):103-13. doi: 10.1590/51415-52 731999000100009 . 
40. Analoni JA. Situação de trabalho dos nutricionistas em empresas de refeições coletivas de Minas Gerais: trabalho técnico, supervisão ou gerência?. Rev Nutr. 1999; 12(3):241-60. doi: 10.1590/S1415-5273199 9000300005.

41. Amorim MMA, Junqueira RG, Jokl L. Adequação nutricional do almoço self-service de uma empresa de Santa Luzia, MG. Rev Nutr. 2005; 18(1):145-56. doi: 10.1590/S1415-52732005000100013.

42. Sousa AA, Proença RPC. Tecnologias de gestão dos cuidados nutricionais: recomendações para qualificação do atendimento nas unidades de alimentação e nutrição hospitalares. Rev Nutr. 2004; 17(4): 425-36. doi: 10.1590/\$1415-52732004000400 003.

43. Assis AMO, Santos SMC, Freitas MCS, Santos JM, Silva MCM. O Programa Saúde da Família: contribuições para uma reflexão sobre a inserção do nutricionista na equipe multidisciplinar. Rev Nutr. 2002; 15 (3): 255-66. doi: 10.1590/S1415-527320 02000300001.

44. Pádua JG, Boog MCF. Avaliação da inserção do nutricionista na Rede Básica de Saúde dos municípios da Região Metropolitana de Campinas. Rev
Nutr. 2006; 19 (4):413-24. doi: 10.1590/S1415-52 732006000400001.

45. Conselho Federal de Nutricionistas. Resolução CFN $N^{\circ} 358 / 2005$. Dispõe sobre as atribuições do Nutricionista no âmbito do Programa de Alimentação Escolar (PAE) e dá outras providências. [acesso 2009 ago. 22]. Disponível em: <ftp://ftp.fnde.gov.br/web/ resolucoes_2005/res358_2005_cfn.pdf>.

46. Ministério da Saúde. Portaria MS n 154, de 24 de Janeiro de 2008. Cria os Núcleos de Apoio à Saúde da Família. [acesso 2009 ago. 22]. Disponível em: $<$ http://dtr2004.saude.gov.br/dab/docs/legislacao/ portaria154_24_01_08.pdf>.

47. Bellotto ML, Linares IP. Las competencias profesionales del nutricionista deportivo. Rev Nutr. 2008; 21(6):633-46. doi: 10.1590/\$1415-527320 08000600003.

48. Brasil. Ministério da Educação. Diretrizes curriculares nacionais do curso de graduação em nutrição. [acesso 2009 ago. 23]. Disponível em: <http:// portal.mec.gov.br/cne/arquivos/pdf/Nutr.pdf>.

Recebido em: 13/11/2009

Aprovado em: 16/12/2010 
